Discrete Comput Geom 27:293-301 (2002)

DOI: $10.1007 / \mathrm{s} 00454-001-0071-6$

\title{
Equipartition of Two Measures by a 4-Fan*
}

\author{
I. Bárány ${ }^{1}$ and J. Matoušek ${ }^{2}$ \\ ${ }^{1}$ Rényi Institute of Mathematics, Hungarian Academy of Sciences, \\ P.O. Box 127, 1364 Budapest, Hungary \\ barany@math-inst.hu \\ and \\ Department of Mathematics, University College London, \\ Gower Street, London WC1E 6BT, England \\ ${ }^{2}$ Department of Applied Mathematics and \\ Institute of Theoretical Computer Science (ITI), Charles University, \\ Malostranské nám. 25, 11800 Praha 1, Czech Republic \\ matousek@kam.mff.cuni.cz
}

\begin{abstract}
We show that, given two probability measures in the plane, there exists a 4-fan that simultaneously equipartitions them. In other words, there is a point and four half-lines emanating from it such that each of the four sectors have measure $\frac{1}{4}$ in both measures.
\end{abstract}

\section{Introduction}

This is a continuation of our previous work [BM] whose terminology and notations are used here without much change. A point $x$ in the plane and four half-lines, $\ell_{1}, \ell_{2}, \ell_{3}, \ell_{4}$, starting from $x$ form a 4-fan. The half-lines are in clockwise order around $x$. They determine four angular sectors $\sigma_{1}, \ldots, \sigma_{4}$ with $\sigma_{i}$ between $\ell_{i}$ and $\ell_{i+1}$. Assume $\lambda$ and $\mu$ are nice probability measures on $\mathbf{R}^{2}$, say, none of them is positive on any line. We showed in $[\mathrm{BM}]$ that there is a 4-fan with $\lambda\left(\sigma_{i}\right)=\mu\left(\sigma_{i}\right)=\frac{1}{5}$ for $i=1,2,3$. (Then, of course, $\lambda\left(\sigma_{4}\right)=\mu\left(\sigma_{4}\right)=\frac{2}{5}$.) Our main result here is

\footnotetext{
* Imre Bárány was supported by Hungarian National Foundation Grants T 032452 and T 029255. Jiři Matoušek was supported by the project LN00A056 of the Ministry of Education of the Czech Republic and by Charles University Grants Nos. 158/99 and 159/99. Part of this research was done during a visit to the University College London and was supported by an EPSRC research fellowship.
} 
Theorem 1.1. Assume $\lambda$ and $\mu$ are absolutely continuous (with respect to the Lebesgue measure) Borel probability measures on $\mathbf{R}^{2}$. Then there is a 4-fan simultaneously equipartitioning both, i.e., $\lambda\left(\sigma_{i}\right)=\mu\left(\sigma_{i}\right)=\frac{1}{4}$ for $i=1,2,3,4$.

It is more convenient to lift the measures and the 4 -fans from $\mathbf{R}^{2}$ to the 2 -sphere $S^{2}$. So let $S^{2}$ be the unit sphere of $\mathbf{R}^{3}$ and let $\mathbf{R}^{2}$ be embedded in $\mathbf{R}^{3}$ as the horizontal plane tangent to $S^{2}$ (at the South Pole). Denote by $\pi$ the central projection from the lower hemisphere to the embedded $\mathbf{R}^{2}$. Clearly, $\pi^{-1}$ lifts any Borel measure on $\mathbf{R}^{2}$ to a Borel measure on the lower hemisphere of $S^{2}$. A 4-fan in $\mathbf{R}^{2}$ is lifted to a 4-fan in $S^{2}$ in a natural way: a spherical 4-fan is a point $x \in S^{2}$ and four great half-circles $\ell_{1}, \ldots, \ell_{4}$ starting at $x$ (and ending at $-x$ ) that are ordered clockwise when we look at them from the origin towards $x$. The angular sector between $\ell_{i}$ and $\ell_{i+1}$ is $\sigma_{i}$. It is clear that a spherical 4-fan is projected by $\pi$ to a 4 -fan in $\mathbf{R}^{2}$. We will prove Theorem 1.1 in a slightly stronger form:

Theorem 1.2. Assume $\lambda$ and $\mu$ are absolutely continuous (with respect to the Lebesgue measure) Borel probability measures on $S^{2}$. Then there is a spherical 4-fan simultaneously equipartitioning both, i.e., $\lambda\left(\sigma_{i}\right)=\mu\left(\sigma_{i}\right)=\frac{1}{4}$ for $i=1,2,3,4$.

In fact, this theorem holds under the weaker assumption that neither $\lambda$ nor $\mu$ is positive on a great circle. This follows from a simple compactness argument.

We call a measure nice if it is a Borel measure, it is a probability measure, it is absolutely continuous with respect to the Lebesgue measure, and it is positive on every nonvoid open set. We prove Theorem 1.2 assuming that both measures are nice. This will suffice for the general case by the same compactness argument. By the same token it is enough to prove the theorem for a dense set of nice measures, and we assume, in case of need, that our measures satisfy certain extra properties.

\section{Equivariant Topology}

Write $V=\left\{(x, y) \in S^{2} \times S^{2}: x \perp y\right\} ; V$ is the Stiefel manifold of orthogonal 2-frames in $\mathbf{R}^{3}$, which is homeomorphic to $\mathrm{SO}(3)$ (see [B]). Of this, we only need that $V$ is three-dimensional and orientable. To every $(x, y) \in V$ we assign the 4-fan $\psi(x, y)=$ $\left(x ; \ell_{1}, \ldots, \ell_{4}\right)$ as follows: $y$ is the midpoint of the great half-circle $\ell_{1}$, and $\ell_{2}, \ell_{3}, \ell_{4}$ are defined by the condition $\lambda\left(\sigma_{i}\right)=\frac{1}{4}$ for all $i$. Further, let $y^{i}$ denote the midpoint of the great half-circle $\ell_{i}(i=1,2,3,4)$, so $y=y^{1}$. Write $X_{4}$ or $X$ for the set of all 4-fans equipartitioning the measure $\lambda$. Then $\psi$ is a map $V \rightarrow X_{4}$.

To evaluate the quality of a 4-fan $\left(x ; \ell_{1}, \ldots, \ell_{4}\right) \in X$ we define a map $\varphi: X \rightarrow \Delta^{3}$ (where $\triangle^{3}$ is the three-dimensional standard simplex: $\Delta^{3}=\left\{x \in \mathbf{R}^{4}: x_{i} \geq 0, \sum_{i=1}^{4} x_{i}=\right.$ 1\}) as $\varphi\left(x ; \ell_{1}, \ldots, \ell_{4}\right)=\left(\mu\left(\sigma_{1}\right), \ldots, \mu\left(\sigma_{4}\right)\right)$. All we want to show is that the map $\varphi \circ \psi: V \rightarrow \triangle^{3}$ takes the value $c=\left(\frac{1}{4}, \frac{1}{4}, \frac{1}{4}, \frac{1}{4}\right)$, the center of the simplex. Assume it does not. Write $\pi$ for the central projection $\triangle^{3} \backslash\{c\} \rightarrow T$, where $T$ is the sphere of radius $\frac{1}{2}$ centered at $c$ in the affine hull of $\Delta^{3}$. Thus $T$, the target space, is topologically an $S^{2}$. (The special choice of the radius $\frac{1}{2}$ will result in simpler notation later.)

We use equivariant topology. The cyclic groups $Z_{4}$ and $Z_{2}$ act on $V, X, \Delta^{3}$ (and, of course, on $T \subset$ aff $\triangle^{3}$ ). The following describes the actions of the generator of $Z_{4}$ on 
$V, X$, and $\triangle^{3}$, denoted by $\omega, \omega^{*}$, and $\omega_{0}$, respectively, and the actions of the generator of $Z_{2}$ on these spaces, denoted by $v, v^{*}$, and $v_{0}$. For $(x, y) \in V$ with $y=y^{1}$, we have

$$
\begin{array}{ll}
\omega\left(x, y^{i}\right)=\left(x, y^{i+1}\right), & v(x, y)=(-x, y), \\
\omega^{*}\left(x ; \ell_{1}, \ldots, \ell_{4}\right)=\left(x, \ell_{2}, \ell_{3}, \ell_{4}, \ell_{1}\right), & v^{*}\left(x ; \ell_{1}, \ell_{2}, \ell_{3}, \ell_{4}\right)=\left(x ; \ell_{4}, \ell_{3}, \ell_{2}, \ell_{1}\right), \\
\omega_{0}\left(t_{1}, t_{2}, t_{3}, t_{4}\right)=\left(t_{2}, t_{3}, t_{4}, t_{1}\right), & v_{0}\left(t_{1}, t_{2}, t_{3}, t_{4}\right)=\left(t_{4}, t_{3}, t_{2}, t_{1}\right) .
\end{array}
$$

It is very easy to see that $\psi \circ \omega=\omega^{*} \circ \psi, \varphi \circ \omega^{*}=\omega_{0} \circ \varphi, \pi \circ \omega_{0}=\omega_{0} \circ \pi$, and the same with $\nu, v^{*}, \nu_{0}$. This shows that the composite map $f=\pi \circ \varphi \circ \psi: V \rightarrow T$ is a $Z_{4}$-map and a $Z_{2}$-map as well: $\omega_{0} \circ f=f \circ \omega$ and $\nu_{0} \circ f=f \circ v$.

Let $G=Z_{2} \times Z_{2}$ (direct product). This $G$ acts on both $V$ and $T$ with generators corresponding to $\omega^{2}$ and $v$ and to $\omega_{0}^{2}$ and $\nu_{0}$. Thus $f: V \rightarrow T$ is a $G$-map. We will show that such a map does not exist if $f$ (or rather $\varphi$ ) comes from two measures.

For later reference we record the actions of $G$ on $V$ and $T$. We rename them as $g_{1}, g_{2}$ corresponding to $\omega^{2}, v$, and $h_{1}, h_{2}$ to $\omega_{0}^{2}$, $\nu_{0}$. Then $g_{3}=g_{1} g_{2}$ and $h_{3}=h_{1} h_{2}$ correspond to the third element of $G$, and with $y=y^{1}$,

$$
\begin{array}{ll}
g_{1}(x, y)=\left(x, y^{3}\right), & h_{1}\left(t_{1}, t_{2}, t_{3}, t_{4}\right)=\left(t_{3}, t_{4}, t_{1}, t_{2}\right) \\
g_{2}(x, y)=(-x, y), & h_{2}\left(t_{1}, t_{2}, t_{3}, t_{4}\right)=\left(t_{4}, t_{3}, t_{2}, t_{1}\right) .
\end{array}
$$

Remark. There is no obstruction to the existence of a $G$-map $f: V \rightarrow T$. An example of such a map is constructed in [BM]. In order to prove Theorem 1.2 we will need to use the fact that $f$ comes from two measures.

\section{An Equivariant Subdivision of $V$}

We are going to construct an equivariant subdivision of $V$. It is going to consist of four three-dimensional domains $D, g_{1} D, g_{2} D, g_{3} D$ and their boundaries. The boundary of $D$ is made up of four two-dimensional cells $P, g_{2} P$ and $Q, g_{1} Q$.

The construction starts with choosing a great circle that halves the measure $\lambda$. We fix the coordinate system so that this great circle coincides with $\left\{x \in S^{2}: x_{1}=0\right\}$. Thus $\lambda(H)=\frac{1}{2}$ where $H$ is the hemisphere $\left\{x \in S^{2}: x_{1} \leq 0\right\}$. We may assume that $\mu(H)=\mu_{0}<\frac{1}{2}$, as otherwise we change the orientation of the first axis or, if $\mu(H)=\frac{1}{2}$, we change the measure $\mu$ a little and use a limiting argument in the end.

For $x \in S^{2} \backslash\left\{ \pm e_{3}\right\}$ let $y^{1}(x)$ be the (unique) unit vector orthogonal to $x$ with the maximum $x_{3}$-coordinate among the unit vectors $y \perp x$. Further, for $i=2,3,4, y^{i}(x)$ is defined by $\omega^{i-1}\left(x, y^{1}(x)\right)=\left(x, y^{i}(x)\right)$. It is easy to check that $y^{1}(x)=y^{1}(-x)$, $y^{3}(x)=y^{3}(-x), y^{2}(x)=y^{4}(-x)$, and $y^{4}(x)=y^{2}(-x)$. Also, for $x \in S^{2}$ with $x_{1}=0$, we have $y^{3}(x)=-y^{1}(x)$.

We need one more piece of notation before we can construct the equivariant subdivision of $V$. Assume $v_{1}, v_{2}, v_{3} \in S^{2}$ are distinct and orthogonal to $x \in S^{2}$. Then $\left\langle v_{1}, v_{2}, v_{3}\right\rangle$ denotes the closed arc of the great circle orthogonal to $x$ lying between $v_{1}$ and $v_{3}$ and containing $v_{2}$. Define $D$ as the closure of the set

$$
\left\{(x, y) \in V: x_{1}>0 \text { and } y \in\left\langle y^{4}(x), y^{1}(x), y^{2}(x)\right\rangle\right\} .
$$




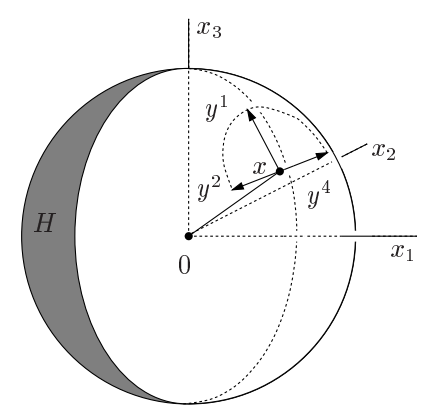

Fig. 1. The domain $D$.

In fact $D$ is just the union of

$$
\left\{(x, y) \in V: x_{1} \geq 0, x \neq \pm e_{3} \text { and } y \in\left\langle y^{4}(x), y^{1}(x), y^{2}(x)\right\rangle\right\}
$$

and the two circles $\left\{\left( \pm e_{3}, y\right) \in V\right\}$. This definition is illustrated in Fig. 1 (which is made for $\lambda$ being the uniform measure on $S^{2}$ ). Further, clearly, the sets $g_{1} D, g_{2} D$, and $g_{3} D$, respectively are the closures of the sets

$$
\begin{aligned}
& \left\{(x, y) \in V: x_{1}>0 \text { and } y \in\left\langle y^{2}(x), y^{3}(x), y^{4}(x)\right\rangle\right\}, \\
& \left\{(x, y) \in V: x_{1}<0 \text { and } y \in\left\langle y^{4}(-x), y^{1}(-x), y^{2}(-x)\right\rangle\right\}, \\
& \left\{(x, y) \in V: x_{1}<0 \text { and } y \in\left\langle y^{2}(-x), y^{3}(-x), y^{1}(-x)\right\rangle\right\} .
\end{aligned}
$$

Thus $D, g_{1} D, g_{2} D, g_{3} D$ have pairwise disjoint interiors and their union covers $V$.

Remark. We mention without proof (since we will not use it) that the following holds. Assume $\lambda=\lambda_{0}$, the uniform probability measure on $S^{2}$. Then clearly $D=\{(x, y) \in$ $\left.V: x_{1} \geq 0, y_{3} \geq 0\right\}$, and one can show directly that $D$ is homeomorphic to $B^{3}$, the unit ball of $\mathbf{R}^{3}$, with $e_{1}$ and $-e_{1}$ identified and $e_{2}$ and $-e_{2}$ identified. This also holds for all nice measures $\lambda$ on $S^{2}$ as well because the homotopy $\lambda_{t}=(1-t) \lambda_{0}+t \lambda$ induces a homotopy between $D\left(\lambda_{0}\right)$ and $D(\lambda)$.

It is easy to see that $\partial D$ consists of four pieces; the first two are $P$ and $Q$ where $P$ is the closure of the set

$$
\left\{(x, y) \in V: x_{1}=0, x_{2}<0 \text { and } y \in\left\langle y^{4}(x), y^{1}(x), y^{2}(x)\right\rangle\right\},
$$

and $Q$ is the closure of the set

$$
\left\{(x, y) \in V: x_{1}>0 \text { and } y=y^{4}(x)\right\}
$$

see Fig. 2. The other two pieces are $g_{2} P, g_{1} Q$.

Note that if $x \in S^{2}$ (with $x_{1}=0, x_{2}<0$ ) tends to $e_{3}$, then $y^{i}(x)$ tends to a limit which we denote by $y^{i}=y^{i}\left(e_{3}\right)(i=1,2,3,4)$. Of course, $y^{1}=e_{2}$ and $y^{3}=-e_{2}$. We also define $y^{i}\left(-e_{3}\right)=y^{i}$. Write $v=\left(e_{3}, y^{4}\right)$ and set $v_{i}=g_{i} v$. We define onedimensional cells $E$ and $F$ of the subdivision: $E=\left\{\left(e_{3}, y\right): y \in\left\langle y^{4}, y^{1}, y^{2}\right\rangle\right\}$ and 

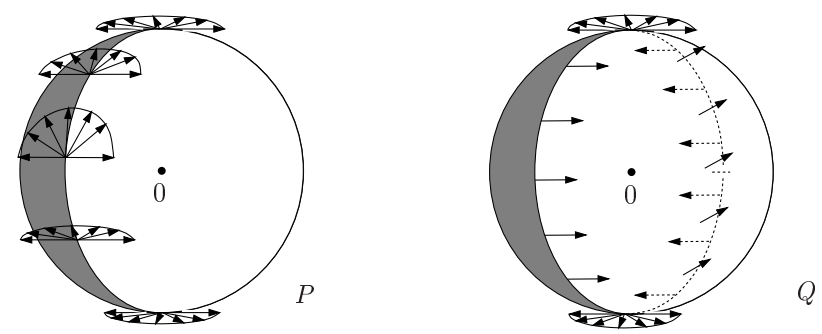

Fig. 2. The 2-cells $P$ and $Q$ in $V$.

$F=\left\{\left(x, y^{4}(x)\right): x_{1}=0, x_{2} \leq 0\right\}$ with $E$ oriented from $v$ to $v_{1}$ and $F$ from $v_{2}$ to $v$; see Fig. 3. (Note that $F$ is well defined even at $x= \pm e_{3}$.)

One can now check that the boundaries of $P$ and $Q$ can be written as $\partial P=E+$ $g_{1} F^{*}+g_{3} E+F$ and $\partial Q=F^{*}+g_{2} E+g_{3} F^{*}+E^{*}$, where $E^{*}$ and $F^{*}$ denote $E$ and $F$ equipped with opposite orientation. Then $\partial g_{2} P$ and $\partial g_{1} Q$ can be computed, and the condition $\partial \partial D=0$ implies that $\partial D=P+Q+g_{2} P^{*}+g_{1} Q^{*}$, where, again, $P^{*}$ and $Q^{*}$ are $P$ and $Q$ with opposite orientation.

The map $f: V \rightarrow T$ is an equivariant $G$-map, and its restrictions $\left.f\right|_{D}$ to $D$ and $\left.f\right|_{\partial D}$ to $\partial D$, respectively, are also equivariant in the sense that, for $i=1,2, f g_{i}(u)=h_{i} f(u)$ whenever $u, g_{i}(u) \in D$ or $u, g_{i}(u) \in \partial D$. More interesting (but equally trivial) is the fact that if $f: D \rightarrow T$ is an equivariant map in the same sense, then it can be extended, and in a unique way, to an equivariant $f: V \rightarrow T$. Simple as it may be, the following observation (see Lemma 1 on page 28 in $[\mathrm{M}]$ ) is crucial.

Lemma 3.1. The restriction $\left.f\right|_{\partial D}: \partial D \rightarrow T$ is of degree zero.

Proof. Note first that $V$, which is $\mathrm{SO}(3)$, is three-dimensional and orientable [B], so $D \subset V$ (which is also three-dimensional and connected) is also orientable.

By changing the measures a little if necessary we may assume $f: D \rightarrow T$ is a differentiable map, and $t \in T$ is a regular value of $f$; that is, its inverse image consists of finitely many pairwise disjoint one-dimensional manifolds. Each such manifold is either a closed Jordan curve (which does not contribute to the degree of $\left.f\right|_{\partial D}$ ) or a Jordan curve

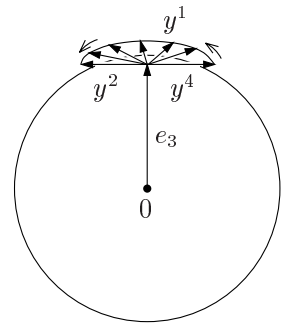

E

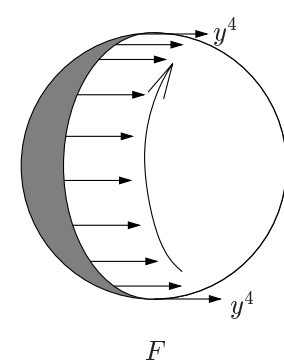

F

Fig. 3. The edges $E$ and $F$. 
connecting two boundary points. Since $D$ is orientable, each such Jordan curve adds +1 at one endpoint, and -1 at the other endpoint, to the degree count. Thus the degree is indeed zero.

Now for Theorem 1.2 it suffices to show

Theorem 3.2. If $f: D \rightarrow T$ comes from two nice measures, then the degree of $\left.f\right|_{\partial D}$ is odd.

We prove this in the last section.

\section{The Winding Number}

Define $b=\left(\frac{1}{2}, 0, \frac{1}{2}, 0\right) \in T$ and $b^{*}=\left(0, \frac{1}{2}, 0, \frac{1}{2}\right) \in T$, and let $L$ denote the line in $\operatorname{aff}\left(\triangle^{3}\right)$ passing through $b$ and $b^{*}$; see Fig. 4. (This is where the special choice of the radius of $T$ is used: $b$ and $b^{*}$ are both in $\triangle^{3}$ and $T$.) Assume $\alpha: S^{1} \rightarrow \triangle^{3}$ (where $S^{1}$ is the unit circle) is a map that avoids $L$. Then the winding number $w(\alpha)$ of $\alpha$ is just the number of times the image $\alpha(s)$ winds around $L$ as $s$ runs through $S^{1}$. This number is taken with orientation; so we choose the broken polygonal path through the vertices (in this order) $(1,0,0,0),(0,1,0,0),(0,0,1,0),(0,0,0,1),(1,0,0,0)$ of $\Delta^{3}$ to have winding number +1 .

Let $L^{\perp}$ be the plane, in the affine hull of $\triangle^{3}$, orthogonal to $L$ and passing through the center $c \in \triangle^{3}$. Clearly, $w(\alpha)$ is the same as the ordinary winding number (around $c$ ) of the mapping $p \circ \alpha: S^{1} \rightarrow L^{\perp}$, where $p$ denotes orthogonal projection to $L^{\perp}$.

Let $B$ be a homeomorphic copy of the unit disk in $\mathbf{R}^{2}$; so $\partial B$ is an $S^{1}$. Assume that $\alpha: B \rightarrow T$ is a map such that the restriction $\left.\alpha\right|_{\partial B}$ avoids $L$. Suppose $\alpha$ is differentiable and that $t \in T$ is a regular point (in the sense of Sard's lemma, see [M] or [L]). Then the signed number of solutions to $\alpha(x)=t$ is denoted by $N(\alpha, t)$, or by $N(\alpha, t, B)$ if we want to specify which $B$ the solutions come from. $N(\alpha, t)$ is just the sum of the signs of the Jacobian of $\alpha$ at the solutions $\alpha(x)=t$. It is well known and actually easy to check that, under the given conditions,

$$
N(\alpha, b, B)-N\left(\alpha, b^{*}, B\right)=w\left(\left.\alpha\right|_{\partial B}\right)
$$

provided $b$ and $b^{*}$ are regular values of $\alpha$.

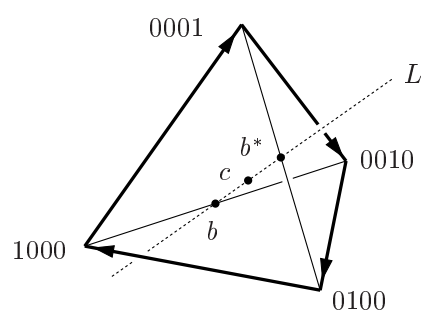

Fig. 4. The winding number with respect to $L$. 
We want to use this statement for $\alpha=\left.f\right|_{P+Q}$. We may assume that $\left.f\right|_{\partial(P+Q)}$ avoids $b$ and $b^{*}$, for otherwise we modify the second measure a little (see Remark 4.4 at the end of this section).

Lemma 4.1. $w\left(\left.f\right|_{\partial(P+Q)}\right)$ is odd.

Proof. Consider $P+Q$ which is a piece of $\partial D$. Its boundary can be expressed as $\partial(P+Q)=g_{1} F^{*}+g_{3} F^{*}+g_{3} E+g_{2} E$. This is just the sum of two cycles $C_{1}+C_{2}$ where

$$
\begin{aligned}
& \left.C_{1}=\left\{(x, y) \in V: y=y^{2}(x)\right) \text { and } x_{1}=0\right\}=g_{1} F^{*}+g_{3} F^{*}, \quad \text { and } \\
& C_{2}=\left\{(x, y) \in V: x=-e_{3}\right\}=g_{2} E+g_{3} E .
\end{aligned}
$$

Clearly, $w\left(\left.f\right|_{\partial(P+Q)}\right)=w\left(\left.f\right|_{C_{1}}\right)+w\left(\left.f\right|_{C_{2}}\right)$.

Claim 4.2. $w\left(\left.f\right|_{C_{1}}\right)=0$.

Proof. To see this, note that, for $(x, y) \in C_{1}$, with $\psi(x, y)=\left(x ; \ell_{1}, \ldots, \ell_{4}\right)$, the union of the first and last sectors is the hemisphere $H=\left\{x \in S^{2}: x_{1} \leq 0\right\}$. So by the choice of the coordinate system (at the start of Section 3), $\mu\left(\sigma_{1}\right)+\mu\left(\sigma_{4}\right)=\mu(H)=\mu_{0}<\frac{1}{2}$. Thus all values of $\varphi$ on $C_{1}$, and consequently all values of $f$ on $C_{1}$, are separated by a plane from $L$. (This proof also shows that $\left.f\right|_{C_{1}}$ avoids the line $L$.)

Claim 4.3. $w\left(\left.f\right|_{C_{2}}\right)$ is odd.

Proof. The cycle $C_{2}$ is an $S^{1}$, the tangent unit circle to $S^{2}$ at $-e_{3}$. Thus the composite map $p \circ f: C_{2} \rightarrow L^{\perp}$ is just an $S^{1} \rightarrow S^{1}$ map. This is a $Z_{2}$ map since $h_{1} \circ f=f \circ g_{1}$ and, as it is very easy to check, the projection $p: T \rightarrow L^{\perp}$ also commutes with $h_{1}$, the corresponding $Z_{2}$-action. Now one form of Borsuk's theorem (one-dimensional version) says that every $Z_{2}$-map $S^{1} \rightarrow S^{1}$ has odd degree. This implies the claim.

The two claims prove Lemma 4.1.

Remark. Alternatively, one can show that $w\left(\left.f\right|_{C_{2}}\right) \equiv 1 \bmod 4$, which is even more than needed for Lemma 4.1. To prove this one observes first that $p \circ f: S^{1} \rightarrow S^{1}$ is a $Z_{4}$-map whose action is $\omega$ and then uses a special case of a theorem from [KZ] (see also [BSS] for a simple proof). The special case says that the degree of an $S^{1} \rightarrow S^{1}$ $Z_{q}$-map is $1 \bmod q$.

Remark 4.4. Here is a sketch of the proof that $\left.f\right|_{\partial(P+Q)}$ avoids $b$ and $b^{*}$, provided that $\mu$ is chosen suitably. As $\partial(P+Q)=C_{1}+C_{2}$ and $\left.f\right|_{C_{1}}$ avoids $L$, it is enough to work with $\left.f\right|_{C_{2}}$. So what we have to achieve by modifying $\mu$ is that $\varphi \circ \psi\left(-e_{3}, y\right)=\left(t_{1}, \ldots, t_{4}\right) \notin L$ for any $\left(-e_{3}, y\right) \in V$. Points on $L$ are characterized by

$$
t_{1}=t_{3}, \quad t_{2}=t_{4}
$$


We assume that $\lambda$ is the normalized Lebesgue measure; the general case goes much the same way. Let $y(s)=(\cos s, \sin s, 0)$, and let $\ell(s)$ be the great half-circle with endpoints $e_{3},-e_{3}$ and midpoint $y(s)$. Write $F(s)$ for the $\mu$-measure of the sector between $\ell(0)$ and $\ell(s) . F(s)$ is a strictly increasing function with $F(0)=0, F(2 \pi)=1$. Write $\varphi \circ \psi\left(-e_{3}, y(s)\right)=t(s)=\left(t_{1}(s), \ldots, t_{4}(s)\right)$. It is readily seen that, for $i=1,2,3,4$,

$$
t_{i}(s)=F\left(s+i \frac{\pi}{2}\right)-F\left(s+(i-1) \frac{\pi}{2}\right)
$$

with the obvious meaning when the argument of $F$ is larger than $2 \pi$. If $t(0) \in L$, then by adding a small mass to $\mu$ near $y(\pi-\varepsilon)$ and subtracting the same little mass near $y(\pi+\varepsilon)$ we can reach $t(0) \notin L$ (here $\varepsilon$ is small). So we may assume that $t(s) \notin L$ in a small vicinity of $s=0$.

By symmetry, it suffices to work with $s \in[0, \pi / 2]$. We can assume next that there are only finitely many solutions to $t_{1}(s)=t_{3}(s)$ by approximating $\mu$ with a measure for which $F(s)$ is a polynomial for $s \in[0,2 \pi]$. Now adding a small mass $\eta$ near $y(\varepsilon)$ and subtracting the same mass near $y(\pi+\varepsilon)$ produces a new (nice) measure $\mu_{\eta}$. (Here $\varepsilon$ is even smaller than before.) The set of solutions to $t_{1}(s)=t_{3}(s)$ (for $s \in[0, \pi / 2]$ ) is the same for $\mu$ and $\mu_{\eta}$. Let $s_{0}$ be a fixed solution to $t_{1}(s)=t_{3}(s)$. Then $t_{2}\left(s_{0}\right)=t_{4}\left(s_{0}\right)$ can only hold for a single value of $\eta$. Thus there are only finitely many $\eta$ for which (2) can have a solution for the new measure $\mu_{\eta}$, and so, for all small enough $\eta$, the new measure $\mu_{\eta}$ approximates $\mu$ well, and the corresponding $\left.f\right|_{\partial(P+Q)}$ avoids $b, b^{*}$.

\section{Proof of Theorem 3.2}

By Hopf's theorem (see [M] or [L]) the degree of $\left.f\right|_{\partial D}$ only depends on its homotopy class. We have seen that $\partial D=P+Q+g_{2} P^{*}+g_{1} Q^{*}$. Fix now our map $f$ on $\partial(P+Q)$ so that it avoids $L$. Then the degree of $\left.f\right|_{\partial D}$ is determined by the homotopy classes of $\left.f\right|_{P}$ and $\left.f\right|_{Q}$. By choosing these homotopy classes suitably we may assume that $b, b^{*}$ are regular values of the map $f: \partial D \rightarrow T$.

Set $p=N(f, b, P), q=N(f, b, Q)$ and $p^{*}=N\left(f, b^{*}, P\right), q^{*}=N\left(f, b^{*}, Q\right)$. Observe that, for $u \in P \cup g_{2} P^{*}, f(u)=b$ if and only if $f\left(g_{2}(u)\right)=b^{*}$ and $f(u)=b^{*}$ if and only if $f\left(g_{2}(u)\right)=b$. Similarly, for $u \in Q \cup g_{1} Q^{*}, f(u)=b$ if and only if $f\left(g_{1}(u)\right)=b$, and $f(u)=b^{*}$ if and only if $f\left(g_{1}(u)\right)=b^{*}$ : checking the first statement, say, goes via $f\left(g_{2}(u)\right)=h_{2}(f(u))=h_{2}(b)=b^{*}$. One has to see that the sign of the solution $u_{0}$ to $f(u)=b$ is the same as the sign of the solution $g_{2}\left(u_{0}\right)$ to $f(u)=b^{*}$, and similarly for the other cases. We do so in the remark at the end of this section.

These observations show that $p=N\left(f, b^{*}, g_{2} P^{*}\right), p^{*}=N\left(f, b, g_{2} P^{*}\right)$ and $q=$ $N\left(f, b, g_{1} Q^{*}\right), q^{*}=N\left(f, b^{*}, g_{1} Q^{*}\right)$.

We count the degree of $\left.f\right|_{\partial D}$ in two ways: at $b$ it is $p+q+p^{*}+q$ and at $b^{*}$ it is $p^{*}+q^{*}+p+q^{*}$. This shows that $q=q^{*}$.

Now formula (1) says that

$$
w\left(\left.f\right|_{\partial(P+Q)}\right)=p+q-p^{*}-q^{*}=p-p^{*},
$$

which is odd by Lemma 4.1. Consequently, $\left.\operatorname{deg} f\right|_{\partial D}=p+q+p^{*}+q=p+p^{*}+2 q$ is odd, finishing the proof of the theorem. 
Remark. It suffices to work out the details for the solutions $u_{0}$ to $f(u)=b$ and $g_{2}\left(u_{0}\right)$ to $f(u)=b^{*}$. We start with taking the derivative of $f\left(g_{2}\left(u_{0}\right)\right)=h_{2}\left(f\left(u_{0}\right)\right)$, and then the determinants:

$$
\operatorname{det} f^{\prime}\left(g_{2}\left(u_{0}\right)\right) \operatorname{det} g_{2}^{\prime}\left(u_{0}\right)=\operatorname{det} h_{2}^{\prime}\left(f\left(u_{0}\right) \operatorname{det} f^{\prime}\left(u_{0}\right) .\right.
$$

So it is enough to see that the sign of $\operatorname{det} g_{2}^{\prime}\left(u_{0}\right)$ and of $\operatorname{det} h_{2}^{\prime}\left(f\left(u_{0}\right)\right)$ are equal to one, or, to put it differently, $g_{2}$ and $h_{2}$ keep the orientations of $\partial D$ and $T$, respectively. The mapping $g_{2}$ is defined on $P$ and on $g_{2} P^{*}$, and one can check directly that it preserves the orientation. For $h_{2}$, we linearly embed $\triangle^{3}$ in $\mathbf{R}^{3}$ by placing its vertices $(1,0,0,0),(0,1,0,0),(0,0,1,0),(0,0,0,1)$ at $(1,1,1),(-1,-1,1),(1,-1,-1)$, $(-1,1,-1)$. Under this embedding $h_{2}$ is just the reflection in the second axis of $\mathbf{R}^{3}$, so it is a linear map whose matrix is diagonal with entries $-1,1,-1$. So $h_{2}$ keeps the orientation of $T$ (and of $\mathbf{R}^{3}$ ).

\section{Acknowledgment}

We thank an anonymous referee for a thorough reading and helpful comments.

\section{References}

[B] G. Bredon. Topology and Geometry (Graduate Texts in Mathematics 139). Springer-Verlag, Berlin, 1993.

[BM] I. Bárány and J. Matoušek. Simultaneous partitions of measures by $k$-fans. Discrete Comput. Geom., 25 (2001), 317-334.

[BSS] I. Bárány, S. Shlosman, and A. Szücs. On a topological generalization of a theorem of Tverberg. J. London Math. Soc., 23 (1981), 158-164.

[KZ] M. A. Krasnoselsky and P. P. Zabrejko. Geometric Problems of Nonlinear Analysis. Nauka, Moscow, 1975 (in Russian).

[L] N. G. Lloyd. Degree Theory. Cambridge University Press, Cambridge, 1978.

[M] J. W. Milnor. Topology from the Differentiable Viewpoint. The University Press of Virginia, Charlottesville, VA, 1965.

Received June 1, 2001, and in revised form October 4, 2001. Online publication February 28, 2002. 